\title{
PAPER
}

\section{Which CT features help predict outcome after head injury?}

\section{J M Wardlaw, V J Easton, P Statham}

J Neurol Neurosurg Psychiatry 2002;72:188-192

See end of article for authors' affiliations

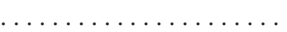

Correspondence to: Dr JM Wardlaw

Department of Clinical

Neurosciences, Western

General Hospital, Crewe

Road, Edinburgh EH4 2XU,

UK:

jmw@skull.dcn.ed.ac.uk

Received

1 February 2001

In revised form 15 August

2001

Accepted 30 August 2001

\begin{abstract}
Background: Information collected at baseline can be useful in predicting patient outcome after head injury. The appearance of the CT brain scan may add useful baseline information. The aim of this study was to evaluate which features on the admission CT scan might add significantly to other baseline clinical information for predicting survival in patients with head injury.

Methods: Baseline CT scans were reviewed for patients with all grades of traumatic head injury in a head injury registry, in which baseline demographic and injury status and outcome at 1 year were recorded. Details from the $\mathrm{CT}$ scan on haemorrhage, brain swelling, and focal or diffuse damage were noted blind to clinical or outcome information and the scans classified according to the simple seven point grading (normal, mild, moderate, or severe focal injury, mild, moderate, or severe diffuse injury). An existing CT scoring system, the trauma coma databank (TCDB) classification, was also used. Logistic regression modelling was used to test the value of the CT appearance, in addition to the other baseline clinical characteristics, in predicting survival at 1 year.

Results: $425 \mathrm{CT}$ scans were read from patients with all severities of injury. Significant independent outcome predictors were age, Glasgow coma score (GCS), pupil reaction, presence of subarachnoid blood, and the simple grading of the overall appearance of the scan (all $p<0.001$ ). The TCDB classification was not a significant predictor of outcome.

Conclusion: Age, GCS, and pupil reaction were all previously shown to be significant predictors of patient survival after head injury. A further two, easy to identify, CT scan variables are independent prognostic variables, and might help to identify patients at high risk of death at the time of admission.
\end{abstract}

n patients with traumatic brain injury, previous studies have examined the association between abnormalities on the CT brain scan, injury severity, and outcome. ${ }^{1-4}$ Others have assessed whether the CT scan might help predict outcome, and suggested methods of classifying the scans-for example, the trauma coma databank (TCDB) classification described in 1991 by Marshall et al, ${ }^{5}$ and three others. ${ }^{6-8}$ Further details of the TCDB or Marshall classification were explained in several publications referring to the data set from which the classification was originally derived, ${ }^{9}{ }^{10}$ and its ability to predict death but not functional recovery was independently tested in one moderate sized study. ${ }^{11}$ The other classifications have not been independently validated.

There are several problems with the previous classifications which limit their generalisability. Most of these classifications were derived in patients with severe head injury $(\mathrm{GCS}<8)$, except for one. ${ }^{7}$ Therefore their value in the generality of patients with head injury admitted to hospital,- that is, all severities requiring admission-has not been tested. In the TCDB classification, the categorisation of mass lesions (into "evacuated" or "non-evacuated") depended on knowing what subsequently actually happened to the patient. As patient management could vary between individual neurosurgeons and centres, the focal categorisation of the TCDB classification might be difficult to apply prospectively to guide management. Most of the studies did not state who actually read the scans, or whether the observers were blinded to the clinical status at baseline or on follow up, an important methodological point if the independent prognostic value of the CT appearance were to be determined.

In a previous study of patients with head injury, we performed prognostic modelling with baseline data collected on admission to hospital to improve outcome prediction. ${ }^{12}$ The variables which were independently predictive of 12 month outcome included age (in years at date of admission), Glasgow coma score (GCS), injury severity score (ISS), pupil score (pupil response on admission, post resuscitation) and the CT brain scan result. In this previous study, the CT scan was simply categorised according to whether or not there was a haematoma present using information obtained from the radiologist's report. There was no independent blinded review of the scans, and the classification resulted in patients with diffuse injury (but without a haematoma) being placed in the same category as those with normal scans. Despite that, modelling showed that the CT appearance was an important predictor of survival over and above the clinical baseline characteristics, in that the presence of any haematoma was predictive of a worse outcome. However, as well as not differentiating between normal scans and scans with diffuse brain swelling, this classification did not take account of the size of the haematoma, its position, or its type (contusion, focal parenchymal, subdural, or extradural). In addition, as the CT categorisation used data extracted from the radiologist's report (by any of many radiologists), or the neurosurgical notes, the CT reader was not "blind" or "expert", or even consistent.

We therefore undertook the present study to investigate precisely which features on admission CT might add significantly to baseline admission information in predicting survival, to improve the previously validated clinical prognostic model, ${ }^{12}$ and test the TCDB classification. ${ }^{5}$ We also undertook a systematic literature search to identify any other schemes for classifying CT of patients with head injury.

\section{METHODS}

Patient identification and data preparation

A total of 1131 consecutive patients with all grades of traumatic head injury, admitted to a regional neurosurgical

Abbreviations: $E D H$, extradural haematoma; $S D H$, subdural haematoma; SAH, subarachnoid haemorrhage; GCS, Glasgow coma score; GOS, Glasgow outcome score; TCDB; trauma coma databank; ISS, injury severity score 
centre from 1 January 1989 until 16 July 1996, comprised the head injury registry data base. For these patients, we attempted to retrieve all baseline admission CT scans from the regional hospitals' radiology departments.

A consultant neuroradiologist read the retrieved CT scans blind to all clinical information, and noted (table 1) the time of the scan; the number, site, and size of any lesions (extradural haematoma $(\mathrm{EDH})$, subdural haematoma $(\mathrm{SDH})$, subarachnoid haemorrhage (SAH), parenchymal contusions, white matter lesions (low or high density), basal ganglia lesions); the presence of a depressed fracture or intracranial air; the presence and amount of any midline shift; whether the ventricles and basal cisterns were compressed, dilated, or normal; the TCDB classification; and finally a simple code to describe the overall appearance of the scan. This code included the following options (table 1): normal appearance; mild focal lesion (for example, small contusion in one area of brain only but the rest of the brain normal); medium focal lesion (for example, several contusions in one or two immediately adjacent areas of brain, or small EDH or small SDH but the rest of the brain normal); mild or moderate diffuse injury (several small contusions or haematomas in several non-adjacent areas of the brain but normal appearance elsewhere); massive focal injury (for example, large EDH or SDH or massive contusions or parenchymal haematoma in one area of the brain); or massive diffuse injury (severe generalised swelling of the brain or many contusions or haematomas in multiple areas). If a follow up scan had been done, and any changes on it were also noted-for example, evidence of evacuation of a mass lesion. Survival at 1 year ${ }^{12}$ was used as the clinical outcome measure in the present study, classified according to the Glasgow outcome scale $(\mathrm{GOS})$, where $\mathrm{l}=$ dead, $2=$ permanent vegetative state, $3=$ severely disabled, $4=$ moderately disabled, $5=$ independent.

\section{Data analyses}

We extracted the baseline demographic details of the patients whose scans had been retrieved and reread from the original head injury database. ${ }^{12}$ To assess the generalisiblity of our results we compared the demographics of patients whose CT had been found with those of the patients whose CT could not be found using $\chi^{2}$ analysis for categorical variables, or two sample $t$ tests for continuous variables, to test for any systematic bias. $\chi^{2}$ tests were used to investigate any associations between grade of injury or GCS total score on admission with each of the extra features on the reread CT scans. Grade of injury was categorised as severely injured (defined as GCS 3-8 plus individual component contributions of eye score equal to 1 , motor score at most 5 , and verbal score at most 2 ) and minor or moderate injuries (defined as GCS 13-15 and GCS 9-12 or GCS 8 if eye score was $>1$, motor score $>5$, and verbal score $>2$ ). The GCS was divided into four groups, as is standard practice; GCS 3-5, 6-8, 9-12, and 13-15.

Logistic regression modelling was used to assess the prognostic significance of the features on the reread CT scans and clinical variables. Candidate predictors in the model included baseline demographic variables (age, ISS, GCS, pupil reaction, cause of injury, sex, alcohol status) and simple CT features (haematoma or no haematoma on CT according to the modified Marshall CT classification as recorded in the original head injury database ${ }^{5}$ ), as well as the additional features available from the reread CT scan. Each variable was initially fitted in a univariate model to screen for prognostic importance, and to decide on the functional form of the relation. Multivariate logistic regression modelling was subsequently used to investigate which of these variables independently predicted survival at 12 months. Standard procedures were used to assess global goodness of fit for final models. ${ }^{13}$

\section{Systematic literature search}

We searched MEDLINE and EMBASE from 1966 and 1980 respectively to August 1999 for studies of the classification
Table 1 Summary of key abnormalities sought on reread CT

\begin{tabular}{|c|c|}
\hline Variable & 425 Reread patients \\
\hline \multicolumn{2}{|l|}{ Extradural haematoma (EDH): } \\
\hline Yes & $74(17)$ \\
\hline No & $347(82)$ \\
\hline Missing & 4 \\
\hline \multicolumn{2}{|l|}{ Subdural haematoma (SDH): } \\
\hline Yes & $182(43)$ \\
\hline No & $240(56)$ \\
\hline Missing & 3 \\
\hline \multicolumn{2}{|l|}{ Subarachnoid haemorrhage (SAH): } \\
\hline Yes & $150(35)$ \\
\hline No & $272(64)$ \\
\hline Missing & 3 \\
\hline \multicolumn{2}{|l|}{ Midline shift: } \\
\hline Yes & $182(43)$ \\
\hline No & $241(57)$ \\
\hline Missing & 2 \\
\hline \multicolumn{2}{|l|}{ Third ventricle: } \\
\hline Normal & $264(62)$ \\
\hline Abnormal (squashed) & $159(38)$ \\
\hline Missing & 2 \\
\hline \multicolumn{2}{|l|}{ TCDB classification: } \\
\hline Diffuse I & $55(13)$ \\
\hline Diffuse II & $146(34)$ \\
\hline Diffuse III & $66(16)$ \\
\hline Diffuse IV & $18(4)$ \\
\hline Evacuated mass lesion & 57 (13) \\
\hline Non-evacuated mass lesion & 4 (1) \\
\hline Mass lesion (evacuation uncertain) & 79 (19) \\
\hline Missing & 0 \\
\hline \multicolumn{2}{|l|}{ Overall appearance: } \\
\hline Normal & 52 (12) \\
\hline Mild focal injury & $25(6)$ \\
\hline Medium focal injury & $38(9)$ \\
\hline Mild/moderate diffuse injury & $123(29)$ \\
\hline Massive focal ( \pm diffuse) & $125(29)$ \\
\hline Massive diffuse ( \pm focal) & $62(15)$ \\
\hline Missing & 0 \\
\hline
\end{tabular}

and association of CT appearance after acute head injury with clinical outcome. We used search terms: (1) computer assisted tomography/or CT; (2) 1 and classification. $\mathrm{mp}$ ( $\mathrm{mp}=$ title, abstract, heading word, trade name, manufacturer's name); (3) 2 and (head injury or brain injury or traumatic head injury or traumatic brain injury). mp; (4) limit 3 to English; (5) 3 and outcome. mp; (6) limit 5 to English language. We also checked references in identified papers. Relevant papers were examined by two people and data extracted on the sample size, year of study, CT variables sought, blinding to clinical baseline variables, outcome measure and timing and result of any analysis on the association of CT with outcome, and whether there had been any validation of any scoring system used to read the CT scan. Studies where the sample size was less than 100 were excluded as these would have limited power to identify valid independent prognostic variables.

\section{RESULTS}

A total of 1131 patients were on the head injury database with complete outcome and demographic information, of which 425 of the original baseline CT scans were found and reread blind by the expert neuroradiologist. Most patients were scanned on the day of injury (median time to CT zero days).

Patients whose scans were retrieved were different from those whose scans could not be found. Those whose scans were reread tended to be more severely injured $(57 \%$ as opposed to $35 \%$ had severe grade head injuries, $\mathrm{p}<0.001$ ) with lower GCS $(\mathrm{p}<0.001)$, higher ISS $(\mathrm{p}=0.036)$, and poorer pupil scores on admission $(p<0.001)$. A greater proportion had haematomas according to the original radiologists' reports 
Table 2 Association between Glasgow coma score and key abnormalities sought on reread CT

\begin{tabular}{lll}
\hline Variable & $\chi^{2}$ Test (df) & p Value \\
\hline EDH (yes/no) & $5.160(3)$ & 0.160 \\
SDH (yes/no) & $10.383(3)$ & $0.016^{*}$ \\
SAH (yes/no) & $33.203(3)$ & $<0.001$ * \\
Midline shift (yes/no) & $10.229(3)$ & 0.017 * \\
3rd ventricle (normal/abnormal) & $19.095(3)$ & $<0.001$ * \\
TCDB: & 21.662 & $0.041^{*}$ \\
$\quad$ Diffuse I-IV or focal & $13.149(3)$ & $0.004^{*}$ \\
$\quad$ Focal/diffuse & $32.526(15)$ & $0.005^{*}$ \\
Overall appearance: & $23.114(6)$ & 0.001 * \\
$\quad$ All six categories & $9.263(3)$ & 0.026 * \\
$\quad$ Normal/mild/massive & & \\
$\quad$ Normal/abnormal & & \\
\hline * Statistically significant associations. & &
\end{tabular}

(55\% v 38\%). There were fewer independent survivors (GOS 5) at 12 months in the reread group (39\%) compared with the group not found for rereading $(43 \% ; \mathrm{p}=0.04)$. However there was no significant difference between the two groups in either total survival at 12 months or in the proportion of poor outcomes (GOS 4-5 v GOS 1-3). Also there were no significant differences between the two groups for age (mean 37 years (SD 20)), cause of injury (road accident $43 \%$ ), sex (where a 3:1 male: female ratio was found in the two groups), whether or not alcohol had been consumed, as well as whether or not the patient had taken any drugs (medicinal or recreational).

\section{Relation between the appearance of the CT scan and injury at baseline}

Table 1 summarises the features visible on the 425 reread CT scans. The association between the CT features and GCS (categorised as GCS 3-5, GCS 6-8, GCS 9-12, and GCS 13-15) was very similar to the association of CT features with grade of injury and therefore the detailed results are reported for GCS only (table 2).

Patients with an SDH or SAH, or midline shift, and an abnormal third ventricle had significantly lower GCS scores (and were more severely injured). The TCDB classification was considered as a full six category variable, as well as a more simple focal or diffuse categorisation. The "overall CT appearance" was also considered as a full categorisation (normal; mild focal; mild/moderate diffuse plus minor focal+diffuse; massive focal (assuming some diffuse also) and massive diffuse \pm focal), as well as a simplified normal $/ \mathrm{mild} / \mathrm{massive}$ and simple normal/abnormal categorisations. All were significantly associated with GCS: a focal or the more severe diffuse injuries (diffuse II, III, and IV) for the TCDB classification; and the mild/moderate diffuse, or massive focal and massive diffuse for the "overall CT appearance" had significantly lower GCS scores. The presence of an EDH was not associated with GCS total or the severity of injury on admission.

\section{Relation between the appearance of the baseline CT scan and clinical outcome: univariate analysis}

There were 20 of the 425 patients whose GOS was not recorded, or were lost to follow up at 12 months, of whom nine did have a 24 month outcome recorded and therefore their 12 month survival status could be inferred, resulting in 414 reread patients available for the regression analysis. Survival or death are the only outcomes for which this back projection method can be applied, as for all other states (grades of disability) patients could change between time points.

In the univariate analyses, age, total GCS on admission, and ISS were significant prognostic variables. Both total GCS on admission and ISS were linearly related to outcome (linearly increasing trend for GCS, and a linearly decreasing trend for ISS). Age was modelled as a covariate (zero up to age 50, then equal to age minus 50 thereafter) as in the previously validated model. ${ }^{12}$ Significant positive associations were found between survival at 12 months and pupil score; the presence of an EDH; and focal versus diffuse injury on the TCDB classification. Negative associations were found with grade of injury (classified as severe or minor/moderate); whether or not the patient had been receiving medication; the presence of an $\mathrm{SDH}$ or SAH; midline shift; abnormal third ventricle; diffuse categories III and IV $v$ a focal injury on the TCDB classification; and "overall appearance" of the scan (normal/ mild/moderate versus massive focal or massive diffuse injury).

The patients' sex, alcohol status, cause of injury, and modified Marshall CT classification (haematoma versus no haematoma, previously extracted from the radiologist's report) were not significant prognostic factors for survival at 12 months in the univariate analysis.

\section{Multivariate analysis to identify prognostic variables}

A multivariate model was then constructed to determine what factors independently predicted whether the patients would survive to 12 months after injury. This model took account of the results of the univariate analyses as well as important biological considerations to keep the model as simple yet as effective to use in clinical practice as possible. ${ }^{12}$ Patients with missing values for any of the variables considered were omitted from this analysis (391 of the potential 425 reread patients were therefore available). The contribution to the reduction in deviance of each variable added sequentially to a model containing other independently significant predictors was assessed and a decision of whether to retain that variable in the model made on this basis, as well as its individual $\mathrm{p}$ value. Age, the admission total GCS, and pupil reaction were rarely missing and remained as independent variables in the model. Each of the variables from the reread CT scan were then considered to determine what they could add to this model.

The final best predictive model included age, GCS, pupil reaction, the presence of subarachnoid blood, and the "overall appearance" of the CT scan. The odds ratios (and associated 95\% confidence intervals (95\% CIs)) for predicting survival at 12 months for each significant prognostic variable are summarised in table 3 . These were significant independent positive associations between survival and GCS and pupil reaction; and significant negative associations between survival and increasing age, the presence of subarachnoid blood, and the "overall appearance" of the CT scan (as massive focal or diffuse injury $v$ normal/mild/moderate injury).

\section{Systematic literature search}

The systematic literature search of EMBASE 1980-99 identified 72 references on CT, classification, and head injury (52 English language) and 19 references on CT, classification, head injury, and outcome (13 English language): the corresponding figures for MEDLINE were 26 (17) and nine (5). Of these, four publications (of $n>100$ ) described a novel CT feature recognition or classification ${ }^{5-8}$ and two described further details of the TCDB classification (table 4). ${ }^{10}$ There were nine other publications but they either had sample size less than $100,{ }^{314-16}$ or made no reference to long term outcome, ${ }^{17}$ or were concerned with only one feature of trauma, ${ }^{2}$ or considered only patients with very mild head injury, ${ }^{18}$ or was a duplicate publication, ${ }^{19}$ or had mixed clinical and CT variables in one classification, ${ }^{1}$ and were therefore excluded. The four studies included had a mean sample size of 418 , none stated whether the CT readers were blind to the clinical data, only two used multivariate analyses to correct for association between CT appearance and clinical baseline variables (to test the independent predictive value of CT), and none of the individual classifications described have been validated in a relevant, independent data set. All the included studies only contained patients with an admission GCS of 8 or less, and 
Table 3 Final multivariate logistic model with $p$ values for sequential tests, adding each variable to a model containing all those above it. Odds ratios (ORs) and $95 \%$ confidence intervals $(95 \% \mathrm{Cls})$ for predicting survival at 12 months for 425 reread patients. Estimated $O R>1$ indicates improved outcome, estimated $O R<1$ indicates a poorer outcome

\begin{tabular}{|c|c|c|c|c|}
\hline Term & $\mathrm{p}$ Value & Details & OR & $95 \% \mathrm{Cl}$ \\
\hline Age & $<0.001$ & Piecewise linear & 0.914 & $(0.884$ to 0.946$)$ \\
\hline GCS & $<0.001$ & Linear increasing from GCS3-15 & 1.247 & (1.107 to 1.405$)$ \\
\hline Pupil Reaction & $<0.001$ & $\begin{array}{l}\text { No reaction } \\
\text { One reactive pupil } \\
\text { Two reactive pupils }\end{array}$ & $\begin{array}{l}1 \\
3.272 \\
5.651\end{array}$ & $\begin{array}{l}- \\
(1.318 \text { to } 8.123) \\
(2.616 \text { to } 12.205)\end{array}$ \\
\hline SAH & $<0.001$ & $\begin{array}{l}\text { No } \\
\text { Yes }\end{array}$ & $\begin{array}{l}1 \\
0.487\end{array}$ & $\overline{(0.264 \text { to } 0.897)}$ \\
\hline Overall appearance & $<0.001$ & $\begin{array}{l}\text { Normal/mild/moderate } \\
\text { Massive focal } \\
\text { Massive diffuse }\end{array}$ & $\begin{array}{l}1 \\
0.594 \\
0.281\end{array}$ & $\begin{array}{l}\overline{-} \\
(0.299 \text { to } 1.181) \\
(0.126 \text { to } 0.625)\end{array}$ \\
\hline
\end{tabular}

therefore are not applicable to the broader range of patients with head injury admitted to hospital. For example, although the patients for whom we were able to find and reread the CT scans for the present study were more severely injured than the generality of the patients in our head injury database, there were still 38\% with CGS 9-15 (compared with $43 \%$ of the whole head injury database). Thus our "overall appearance" classification is applicable to all patients with head injury admitted to hospital, not just those of GCS of 8 or less.

\section{DISCUSSION}

We have determined that three easy to identify clinical variables (age, GCS, and pupil reaction) and two easy to identify CT scan variables (presence of SAH and the "overall appearance" of the scan) are independent prognostic variables for survival after head injury. These variables are all readily identifiable at the time of admission allowing an immediate assessment of prognosis to be made. Although abnormalities tend to become more visible on CT if the scan is delayed until a day or more after injury, clinical practice demands information on which to base management decisions immediately.

The present study is the first validation of the TCDB classification in an independent data set in which half the patients were GCS of 8 or less. Although CT features are independently predictive of outcome, the TCDB classification does not capture the predictive information in our study population as closely as the simple classification derived in the present paper. This may be because the TCDB classification was originally devised in severely injured patients only (and therefore does not work on moderate or mildly injured patients) or because it required knowledge of what happened to the patient subsequently (for example, evacuation of a haematoma) so was retrospectively rather than prospectively applied. When applied prospectively (as it is necessary to do in acute clinical practice) as we have, it does not work.

Although attempts were made to obtain all scans, less than $50 \%$ were traced. This is not surprising as the patients with head injury came through six different hospital $x$ ray departments up to 7 years before the present study and moved both within and between departments and hospitals in the course of their care. Often the patient's name was unknown at the time of first scan ("unknown male") leading to a large proportion of scans which could not be identified retrospectively with sufficient reliability to be included. The more seriously affected patients ended up in intensive care and so were perhaps a more captive patient population and therefore the admission scans for these more severely injured patients were more likely to be retrieved. Thus the patients for whom we were able to find and reread the scans had significantly more severe grade head injuries, more visible haematomas, and less patients in this group had two reactive pupils on admission to hospital compared with those patients whose scans were unobtainable for rereading. There were, however, no significant differences in their age, sex, cause of accident, whether or not they had consumed alcohol or drugs, or in their survival at 12 months or the proportion of poor outcomes.

We have shown that an SDH or SAH, midline shift, abnormal third ventricle, TCDB classification, and a description of the "overall appearance" of the scan were associated with the admission GCS. Thus it would be possible to identify these more severely injured patients with worse prognosis by noting these easy to identify features on the CT, and target their management accordingly. The presence of EDH, midline shift, and a TCDB classification simplified to focal or diffuse were not significantly associated with injury severity, however.

In a univariate analysis, significant associations suggested that patients would have improved chances of survival at 12

Table 4 Previous studies of the predictive value of CT in head injury for long term outcome

\begin{tabular}{|c|c|c|c|c|c|c|c|c|c|}
\hline $\begin{array}{l}\text { Study } \\
\text { (1st author) }\end{array}$ & Year & $\begin{array}{l}\text { Years of } \\
\text { study }\end{array}$ & $\mathrm{n}$ & $\begin{array}{l}\text { Blinded } \\
\text { CT? }\end{array}$ & CT scale & $\begin{array}{l}\text { Outcome } \\
\text { time }\end{array}$ & $\begin{array}{l}\text { Significant predictive } \\
\text { variables }\end{array}$ & Independent CT death & $\begin{array}{l}\text { Predictive of: } \\
\text { poor outcome }\end{array}$ \\
\hline Marshall $^{5}$ & 1991 & $\begin{array}{l}01 / 84- \\
09 / 87\end{array}$ & 746 & $\begin{array}{l}\text { Not } \\
\text { stated }\end{array}$ & $\mathrm{TCDB}$ & Discharge & $\begin{array}{l}\text { Age, motor score, CT } \\
\text { score }\end{array}$ & Increased brain swelling & $\begin{array}{l}\text { Increased brain } \\
\text { swelling }\end{array}$ \\
\hline Liu $^{7}$ & 1995 & $\begin{array}{l}08 / 85- \\
07 / 87\end{array}$ & 334 & $\begin{array}{l}\text { Not } \\
\text { stated }\end{array}$ & 0-5 Scale swelling & 12 months & Univariate analysis only & Brain swelling & Brain swelling \\
\hline Fearnside $^{6}$ & 1993 & Not stated & 315 & $\begin{array}{l}\text { Not } \\
\text { stated }\end{array}$ & 9 Features & 6 months & $\begin{array}{l}\text { Age, GCS motor score, } \\
\text { CT features }\end{array}$ & $\begin{array}{l}\text { Brain swelling, midline } \\
\text { shift, IVH }\end{array}$ & $\begin{array}{l}\text { Contusion, } \mathrm{ICH} \text {, } \\
\text { SAH }\end{array}$ \\
\hline Lobato $^{8}$ & 1983 & $\begin{array}{l}11 / 77 \text { * } \\
07 / 82\end{array}$ & 277 & $\begin{array}{l}\text { Not } \\
\text { stated }\end{array}$ & $\begin{array}{l}8 \text { Categories } \\
\text { diffuse/mass }\end{array}$ & 6 months & Univariate analysis only & SDH, multiple contusions & $\mathrm{n} / \mathrm{s}$ \\
\hline
\end{tabular}

Studies from the same data set as Marshall ${ }^{5}$ are not included; baseline clinical score for entry into all studies was GCS $\leqslant 8$. 
months if they were younger, had higher GCS, lower ISS, and at least one reactive pupil, (further improved if both pupils are reacting) on admission to hospital. Injury severity, classified as severe or minor/moderate, was also a significant predictor where patients with severe head injury were more likely to have a poorer outcome (odds ratio (OR) $0.3295 \%$ CI 0.18 to 0.55 ). However, ISS was not considered at the multivariate stage as it was confounded by total GCS total on admission. Modelling CT diagnosis by a modified Marshall classification (categorising each patient as having a haematoma or not) was not a significant univariate predictor of survival, but was still considered at the multivariate stage due to its importance as the only available CT variable in the previously validated model. ${ }^{12}$ There was a trend towards improved survival in patients with "no haematoma" (OR 1.31, 95\% CI 0.80 to 2.13 ) corroborating the previously validated model in a similar group of patients. ${ }^{12}$

In addition to these clinical variables, we have investigated which extra features from a CT scan might be helpful in predicting survival after traumatic head injury. We have shown that modelling CT diagnosis by TCDB classification (categorising each patient as having either a focal injury, or four categories of diffuse injury) was a significant predictor in the univarate analysis of survival at 12 months, whether as the full categorisation (diffuse I-IV compared with a focal injury), or as a focal versus diffuse classification. The results of this particular univariate analysis were interesting in that it suggests that those patients with a diffuse injury I or diffuse injury II will do better at 12 months after injury than those with a focal injury, (ORs 4.89 and 3.20 respectively; corresponding 95\% CIs 1.65 to 14.47 and 1.66 to 6.15$)$. Patients with diffuse injury IV were significantly worse off (OR $0.34,95 \%$ CI 0.12 to 0.95 ) with a similar, but non-significant effect if they were diffuse injury III, (OR 0.93, 95\% CI 0.48, 1.78). Reducing this variable to two categories in the univariate analysis, focal or diffuse injury, we found a positive significant association between survival and diffuse injury for this group of patients, as compared with focal injury, whether evacuated or not (OR 1.88, $95 \%$ CI 1.15 to 3.06 ). However, the TCDB classification did not remain a significant independent outcome predictor in the multivariate model once clinical features were included.

The presence of subarachnoid blood and the new suggested classification of the "overall appearance" of the scan seems to give a simpler and more intuitive feel of what might be occurring in that there is a clearer separation of types of injury in the regression analysis as well as allowing a "normal" scan option. No significant difference was found for survival at 12 months if the abnormality on CT scan was "mild focal", "medium focal", or "mild/moderate diffuse" compared with a "normal" scan so these four categories were merged, resulting in significant negative associations between this new reference category and massive focal injury (assuming some diffuse), and massive diffuse injury (assuming some focal) (OR 0.30 and 0.14 respectively, with corresponding 95\% CIs 0.17 to 0.53 and 0.07 to 0.27 ). This suggests that there is little point in worrying about recognising minor or subtle abnormalities on the scan in terms of outcome prediction as these are not predictive of poor outcome over and above the basic clinical state of the patient. Obviously, recognition of surgically remediable abnormalities such as extradural or subdural haematomas is important for acute patient management. Recognition of massive diffuse or focal injury does indicate a worse prognosis than would have been predicted by the clinical features alone and should alert the medical staff (as known intuitively) to the likelihood of very poor outcome.

Final models in a multivariate regression analysis show similar significant baseline clinical predictors for this group of reread patients, with the same direction of effect on survival, as for the previously validated predictive model. ${ }^{12}$ We have therefore confirmed that age, admission GCS, and pupil reaction are all independent significant predictors of survival, and shown that a new "overall appearance" variable from the CT scan, is independently significant over and above these baseline factors in predicting survival, together with the presence of subarachnoid blood.

It would seem that this new assessment of a scan would be indicative of survival at 12 months, and although the interobserver variability of reading the CT scans remains to be tested, this classification system would be simple and easy to apply in routine practice. It highlights specific features which admitting doctors should seek on the admission CT. It could be added to the prognostic nomogram derived in previous study ${ }^{12}$ and therefore used to triage patients effectively.

\section{ACKNOWLEDGEMENTS}

The Head Injury Study Registry was established by the late Professor JD Miller. We thank the radiographers and research staff who helped retrieve the CT scans. The study was funded by the UK Medical Research Council Special Project Grant G9508752 and the Clinical Research Initiative in Clinical Neurosciences Grant Nos G9301975 and G9301951. Sources of funding for this work were the UK Medical Research Council Special Project Grant G9508752 and the Clinical Research Initiative in Clinical Neurosciences Grant Nos G9301975 and G9301951.

Authors' affiliations

J M Wardlaw, V J Easton, P Statham, Department of Clinical Neurosciences, Western General Hospital, Edinburgh, UK

\section{REFERENCES}

1 Gennarelli TA, Spielman GM, Langfitt TW, et al. Influence of the type of intracranial lesion on outcome from severe head injury. A multicentre study using a new classification system. J Neurosurg 1982;56:26-32.

2 Greene KA Marciano FF, Johnson BA, et al. Impact of traumatic subarachnoid hemorrhage on outcome in nonpenetrating head injury. $J$ Neurosurg 1995;83:445-52.

3 Kido DE, Cox C, Hamill RW, et al. Traumatic brain injuries: predictive usefulness of CT. Radiology 1992;182:777-81.

4 Teasdale G, Teasdale E, Hadley D. Computed tomographic and magnetic resonance imaging [review]. J Neurotrauma 1992;(suppl 9):S249-57.

5 Marshall LF, Marshall SB, Klauber MR, et al. A new classification of head injury based on computerised tomography. J Neurosurg 1991:75(suppl):S14-20.

6 Fearnside MR, Cook RI, McDougall P, et al. The Westmead head injury project outcome in severe head injury: a comparative analysis of pre-hospital, clinical and CT variables. Br J Neurosurg 1993;7:267-79.

7 Liu HM, Tu YK, Su CT. Changes of brainstem and perimesencephalic cistern: dynamic predictor of outcome in severe head injury. Journal of Trauma Injury Infection and Critical Care 1995;38:330-3.

8 Lobato RD, Cordobes F, Rivas JJ, et al. Outcome from severe head injury related to the type of intracranial lesion: a computerised tomography study. J Neurosurg 1983;59:762-74.

9 Aldrich EF, Eisenberg HM, Saydjari C, et al. Diffuse brain swelling in severely head-injured children: a report from the $\mathrm{NIH}$ traumatic coma data bank. J Neurosurg 1992;76:450-4.

10 Eisenberg HM, Gary HE, Aldrich EF, et al. Initial CT findings in 753 patients with severe head injury: a report from the $\mathrm{NIH}$ traumatic coma data bank. J Neurosurg 1990;73:688-698.

11 Teasdale E, Eltabu M, Teasdale G, et al. CT correlation with neuropathology in 104 autopsied cases of head injury. Neuroradiology 1996;38:192.

12 Signorini DF, Andrews FJD, Jones PA, et al. Predicting survival using simple clinical variables: a case study in traumatic brain injury. $J$ Neurology Neurosurg Psychiatry 1999;66:20-5.

13 Hosmer DW, Lemeshow S. Applied logistic regression. New York: John Wiley, 1989

14 Poca MA, Sahuquillo J, Baguena M, et al. Incidence of intracranial hypertension after severe head injury: a prospective study using the traumatic coma data bank classification. Acta Neurochirurgica 1998;(suppl 71):27-30.

15 Raabe A, Grolms C, Keller M, et al. Correlation of computed tomography findings and serum brain damage markers following severe head injury. Acta Neurochirurgica 1998;140:787-91.

16 van der Naalt J, Hew JM, van Zomeren $\mathrm{AH}$, et al. Computed tomography and magnetic resonance imaging in mild to moderate head injury: Early and late imaging related to outcome. Ann Neurol 1999;46:70-8.

17 Lobato RD, Gomez PA, Alday R, et al. Sequential computerized tomography changes and related final outcome in severe head injury patients. Acta Neurochirurgica 1997:139:385-91.

18 Culotta VP, Sementilli ME, Gerold K, et al. Clinicopathological heterogeneity in the classification of mild head injury. J Neurosurg 1996;38:245-50.

19 Marshall LF, Marshall SB, Klauber MR, et al. The diagnosis of head injury requires a classification based on computed axial tomography. $J$ Neurotrauma 1992;9(suppl 1):S287-92. 